\title{
Clinical characteristics and therapeutic outcomes of nosocomial super-infection in adult bacterial meningitis
}

\author{
Chi-Ren Huang ${ }^{1}$, Shu-Fang Chen ${ }^{1,2}$, Cheng-Hsien Lu', Yao-Chung Chuang ${ }^{1}$, Nai-Wen Tsai ${ }^{1}$, Chiung-Chih Chang ${ }^{1,2}$, \\ Hung-Chen Wang ${ }^{3}$, Chun-Chih Chien ${ }^{4}$ and Wen-Neng Chang ${ }^{1 *}$
}

\begin{abstract}
Background: Super-infection in adult bacterial meningitis (ABM) is a condition wherein the cerebrospinal fluid (CSF) grows new pathogen(s) during the therapeutic course of meningitis. It is an uncommon but clinically important condition rarely examined in literature.

Methods: Twenty-seven episodes of super-infection states in 21 ABM patients collected in a 9.5-year study period (January 2001 to June 2010) were evaluated. The clinical characteristics, implicated pathogens, results of antimicrobial susceptibility tests, and therapeutic outcomes were analyzed.

Results: Twenty-one patients (13 men, 8 women) aged 25-73 years (median, 45 years) had post-neurosurgical state as the preceding event and nosocomial infection. The post-neurosurgical states included spontaneous intracranial hemorrhage (ICH) with craniectomy or craniotomy with extra-ventricular drainage (EVD) or ventriculo-peritoneal shunt (VPS) in 10 patients, traumatic ICH with craniectomy or craniotomy with EVD or VPS in 6 patients, hydrocephalus s/p VPS in 2 patients, and one patient each with cerebral infarct s/p craniectomy with EVD, meningeal metastasis s/p Omaya implant, and head injury. All 21 patients had EVD and/or VP shunt and/or Omaya implant during the whole course of ABM. Recurrent fever was the most common presentation and the implicated bacterial pathogens were protean, many of which were antibiotic resistant. Most patients required adjustment of antibiotics after the pathogens were identified but even with antimicrobial therapy, 33.3\% (7/21) died. Morbidity was also high among survivors.

Conclusions: Super-infection in ABM is usually seen in patients with preceding neurosurgical event, especially insertion of an external drainage device. Repeat CSF culture is mandatory for diagnostic confirmation because most of the implicated bacterial strains are non-susceptible to common antibiotics used. Unusual pathogens like anaerobic bacteria and fungi may also appear. Despite antimicrobial therapy, prognosis remains poor.
\end{abstract}

\section{Background}

Super-infection in adult bacterial meningitis (ABM) is a condition in which the cerebrospinal fluid (CSF) grows new pathogen(s) during the therapeutic course of meningitis $[1,2]$. This uncommon situation is rarely examined solely in overview studies of ABM [3,4]. In ABM management, early and appropriate antibiotic use is an important strategy for better outcomes [5-7].

\footnotetext{
* Correspondence: cwenneng@ms19.hinet.net

'Department of Neurology, Kaohsiung Chang Gung Memorial Hospital and

Chang Gung University College of Medicine, Kaohsiung, Taiwan

Full list of author information is available at the end of the article.
}

However, super-infection may cause a dilemma in antibiotic choice because starting a new antimicrobial regimen for this complicated condition during the therapeutic course of ABM is a difficult decision. To better delineate the clinical characteristics of superinfection in ABM, this study analyzed the clinical characteristics and therapeutic outcomes of ABM patients with super-infection.

\section{Methods}

The medical and microbiological records for CSF of all adult patients with bacterial meningitis admitted to

\section{Biomed Central}

(c) 2011 Huang et al; licensee BioMed Central Ltd. This is an Open Access article distributed under the terms of the Creative Commons Attribution License (http://creativecommons.org/licenses/by/2.0), which permits unrestricted use, distribution, and reproduction in any medium, provided the original work is properly cited. 
Chang Gung Memorial Hospital (CGMH)-Kaohsiung, a 2482-bed acute-care teaching hospital and the largest tertiary care center in southern Taiwan, from January 2001 to June 2010 (9.5 years) were retrospectively reviewed. The hospital's Ethics Committee approved the study (IRB 99-1897C).

Diagnosis of ABM was based on: i) age $\geq 17$ years; ii) positive CSF culture in patients with clinical presentation of acute meningitis (i.e. fever, altered consciousness, or seizure); and iii) at least one of the following CSF parameters: leukocyte count $>0.25 \times 10^{9} / \mathrm{L}$ with predominant polymorphonuclear cells; CSF lactate concentration $>3.5 \mathrm{mmol} / \mathrm{L}$; glucose ratio (CSF glucose/serum glucose) $<0.4$; or CSF glucose concentration $<2.5 \mathrm{mmol} /$ $\mathrm{L}$ if no simultaneous blood glucose was determined $[3,4]$. "Super-infection" in ABM was defined as a condition wherein CSF grew new pathogen(s) during the therapeutic course of an existing bacterial meningitis $[1,2]$.

For this study, ABM was classified as either nosocomial or community-acquired. Nosocomial meningitis was defined as positive bacterial infection not present on hospital admission, clinical evidence of infection no sooner than 48 hours after admission, or clinical evidence of meningitis within a short period of time, i.e. within one month after discharge from the hospital where the patient received an invasive procedure, especially a neurosurgical one. Otherwise, the patient was considered as having "community-acquired" infection.

Patients who developed meningitis related to head trauma with skull fractures or neurosurgical procedures were classified as "post-neurosurgical" meningitis. Otherwise, patients who demonstrated no clear distinctive disease characteristics and who had not undergone any invasive procedures were classified as "spontaneous" meningitis. "Mixed-infection" was defined as at least two bacterial organisms isolated concomitantly from CSF culture $[4,8]$.

During the study period, intravenous administration of vancomycin with either a $3^{\text {rd }}$ - or $4^{\text {th }}$ - generation cephalosporin (e.g. ceftriaxone, ceftazidime, or cefepime) were the initial empiric antibiotics used to treat adult patients with clinical evidence of bacterial meningitis. Further antibiotic adjustment was guided by results of pathogen identification and antibiotic susceptibility tests. Antibiotic susceptibility of the isolated pathogen was tested using the Kirby-Bauer diffusion method (BBL, Muller-Hinton II agars; Becton Dickinson Microbiology Systems, Cockeysville, MD) while bacterial susceptibility to antimicrobial agents was determined according to criteria of the National Committee for Clinical Laboratory Standards (CLSI). The therapeutic results were evaluated by using Glasgow Outcome Scale (GOS) [9]: score 1 = death, score 2 = persistent vegetative state, score 3 = severe disability, score $4=$ moderate disability, score $5=$ good recovery. Scores of 1-3 were classified as "poor prognosis" while scores of 4 and 5 were "good prognosis".

For comparative analysis, the patients were divided into fatal and non-fatal groups. Data such as sex, type of infection, underlying conditions, and clinical manifestations between these two patient groups were analyzed by Fisher's exact test. Age between the two groups was analyzed by Mann-Whitney U test.

\section{Results}

During the study period, 21 ABM patients aged 25-73 years (median 45 years), including 13 men and 8 women, had super-infection. Their basic clinical and laboratory data are listed in Tables 1 and 2. All had post-neurosurgical state as the preceding event and had contracted the infection nosocomially. The post-neurosurgical states included spontaneous intracranial hemorrhage (ICH) with craniectomy or craniotomy with extraventricular drainage (EVD) or ventriculo-peritoneal shunt (VPS) in 10 patients (Cases 2-4, 9-11, 14, 17, 20, 21 ), traumatic ICH with craniectomy or craniotomy with EVD or VPS in 6 patients (Cases 5, 6, 12, 13, 16, 18), hydrocephalus s/p VPS in 2 patients (Cases 7, 15), cerebral infarct $s / p$ craniectomy with EVD in Case 1, meningeal metastasis s/p Omaya implant in Case 8, and head injury in Case 19. All 21 patients had undergone EVD and/or VPS and/or Omaya implant during the whole clinical course of ABM. The underlying medical conditions included diabetes mellitus in 3 patients (Cases 3, 10, 20), lung cancer in Case 8, brain tumor in Case 11, and liver cirrhosis in Case 21.

There were 27 episodes of super-infection in the 21 ABM patients. The initial and subsequently implicated pathogens are listed in Table 1. Eight patients (Cases 18 ) were converted from Gram-positive $(\mathrm{G}(+))$ pathogen infection to Gram-negative $(G(-))$ pathogen infection and two (Cases 9 and 10) were converted from G(-) pathogen to $G(+)$ pathogen infection. Six patients (Cases 11-16) were converted from $G(-)$ pathogen infections to another G(-) pathogen infection. Cases 17-21 had other converted states of implicated pathogens, while Case 18 had a more complicated conversion in different CSF cultures with different combinations of implicated pathogens noted in each culture.

Of the 27 episodes of super-infection, the time interval from finding the previous pathogen to detecting a new pathogen, i.e. appearance of super-infection, ranged from 3 to 90 days. Changes in clinical and laboratory evidence of super-infection were noted in 20 of the 27 episodes. Recurrent fever was the most common and found in 17 episodes (Cases 1-3, 5-8, 10, 11, 14-16, 18, $20)$, The other new presentations were CSF pleocytosis in 5 episodes (Cases $1,3,7,19,20$ ), peripheral leukocytosis in 3 episodes (Cases 1, 13, 20), appearance of 
Table 1 Clinical data and implicated pathogens in the super-infections of the study patients with adult bacterial meningitis

\begin{tabular}{|c|c|c|c|c|c|c|c|c|c|c|c|c|}
\hline Case & Gender & $\begin{array}{l}\text { Age } \\
\text { (years) }\end{array}$ & $\begin{array}{l}\text { Underlying } \\
\text { condition }\end{array}$ & $\begin{array}{l}\text { Initial pathogen } \\
\text { (s) }\end{array}$ & Antibiotics & Management & $\begin{array}{l}\text { Interval* }^{*} \\
\text { (days) }\end{array}$ & $\begin{array}{l}\text { New } \\
\text { presentation }\end{array}$ & New pathogen(s) & Antibiotics & Management & Survived \\
\hline \multicolumn{13}{|c|}{ More than 7 days interval* } \\
\hline & \multicolumn{12}{|c|}{$\operatorname{Gram}(+) \rightarrow \mathrm{G}(-)$ or $\mathrm{G}(-) \rightarrow \mathrm{G}(+)$} \\
\hline 1 & M & 55 & $\begin{array}{l}\text { Infarct, craniectomy, } \\
\text { EVD }\end{array}$ & $\begin{array}{l}\text { Staphylococcus } \\
\text { aureus }^{\S}\end{array}$ & LZD+EPM & $\begin{array}{l}\text { Removed EVD } \\
\rightarrow \text { new EVDs }\end{array}$ & 61 & $\begin{array}{l}\text { Fever, } \\
\text { leukocytosis, } \\
\text { CSF pleocytosis }\end{array}$ & ESBL-Escherichia coli & MEP & Removed EVD & No \\
\hline 2 & $\mathrm{~F}$ & 48 & $\begin{array}{l}\text { SICH, craniectomy, } \\
\text { EVD }\end{array}$ & $\begin{array}{l}\text { Staphylococcus } \\
\text { aureus }^{\S}\end{array}$ & $\mathrm{VA}+\mathrm{CAZ}$ & $\begin{array}{l}\text { Removed EVD } \\
\rightarrow \text { craniotomy, } \\
\text { new EVDs }\end{array}$ & 34 & $\begin{array}{l}\text { Fever, } \\
\text { hydrocephalus }\end{array}$ & $\begin{array}{l}\text { Stenotrophomonas } \\
\text { maltophilia" }^{\#}\end{array}$ & $\begin{array}{l}\text { MOX+TMP } \\
+ \text { TIG }+ \\
\text { ATM }\end{array}$ & $\begin{array}{l}\text { Removed EVD } \rightarrow \\
\text { New EVD } \rightarrow \\
\text { removed EVD } \rightarrow \\
\text { VP shunt }\end{array}$ & Yes \\
\hline 3 & $\mathrm{~F}$ & 55 & $\begin{array}{l}\mathrm{DM}, \mathrm{SICH}, \\
\text { craniotomy, VPS }\end{array}$ & $\begin{array}{l}\text { Staphylococcus } \\
\text { aureus }^{\$}\end{array}$ & VA & $\begin{array}{l}\text { Removed VPS, } \\
\text { debridement } \rightarrow \\
\text { new EVD }\end{array}$ & 11 & $\begin{array}{l}\text { Fever, CSF } \\
\text { pleocytosis }\end{array}$ & $\begin{array}{l}\text { Acinetobacter } \\
\text { baumannii }\end{array}$ & LZD+MEP & $\begin{array}{l}\text { New EVD } \rightarrow \\
\text { ventriculo-pleural } \\
\text { shunt }\end{array}$ & Yes \\
\hline 4 & $\mathrm{~F}$ & 41 & $\begin{array}{l}\text { SICH, craniectomy, } \\
\text { EVD }\end{array}$ & $\begin{array}{l}\text { Staphylococcus } \\
\text { epidermidis }\end{array}$ & VA & $\begin{array}{l}\text { Craniotomy, EVD } \\
\rightarrow \text { VPS }\end{array}$ & 47 & No & $\begin{array}{l}\text { Enterobacter cloacae } \\
+ \text { Pseudomonas } \\
\text { aeruginosa }\end{array}$ & CAZ & Removed VPS & No \\
\hline 5 & M & 73 & $\begin{array}{l}\text { TICH, craniectomy, } \\
\text { EVD (removed) }\end{array}$ & $\begin{array}{l}\text { Coagulase- } \\
\text { negative } \\
\text { staphylococci }^{\S}\end{array}$ & VA & New EVD & 9 & $\begin{array}{l}\text { Fever, seizure, } \\
\text { altered } \\
\text { consciousness }\end{array}$ & Enterobacter cloacae & $\mathrm{ROC}$ & $\begin{array}{l}\text { Removed EVD } \rightarrow \\
\text { VP shunt }\end{array}$ & Yes \\
\hline 6 & M & 46 & $\begin{array}{l}\text { TICH, craniectomy, } \\
\text { VPS }\end{array}$ & $\begin{array}{l}\text { Coagulase- } \\
\text { negative } \\
\text { staphylococci }^{\S}\end{array}$ & VA & $\begin{array}{l}\text { Externalisation } \\
\text { VPS } \rightarrow \text { removed } \\
\text { VPS } \rightarrow \text { new } \\
\text { EVDs }\end{array}$ & 46 & Fever & $\begin{array}{l}\text { Coagulase-negative } \\
\text { staphylococci }^{\xi}+ \\
\text { Acinetobacter } \\
\text { baumannii }\end{array}$ & MEP & $\begin{array}{l}\text { Removed EVD } \rightarrow \\
\text { new EVDs } \rightarrow \text { VP } \\
\text { shunt }\end{array}$ & Yes \\
\hline 7 & M & 34 & Hydrocephalus, VPS & $\begin{array}{l}\text { Staphylococcus } \\
\text { epidermidis }\end{array}$ & VA & $\begin{array}{l}\text { Removed VPS, } \\
\text { New EVDs }\end{array}$ & 28 & $\begin{array}{l}\text { Fever, CSF } \\
\text { pleocytosis }\end{array}$ & Acinetobacter spp & CEF & $\begin{array}{l}\text { Removed EVD } \rightarrow \\
\text { New EVD } \rightarrow \\
\text { removed } \rightarrow \text { VPS }\end{array}$ & Yes \\
\hline 8 & $\mathrm{~F}$ & 45 & $\begin{array}{l}\text { Lung cancer, } \\
\text { meningeal } \\
\text { metastasis, Omaya } \\
\text { implant, infection }\end{array}$ & $\begin{array}{l}\text { Coagulase- } \\
\text { negative } \\
\text { staphylococci }\end{array}$ & VA & $\begin{array}{l}\text { Debridement, } \\
\text { removed Omaya } \\
\text { implant }\end{array}$ & 42 & Fever & Acinetobacter spp & CAZ & & Yes \\
\hline 9 & $\mathrm{~F}$ & 48 & $\begin{array}{l}\text { Brain tumor, } \mathrm{SICH} \text {, } \\
\text { craniectomy }\end{array}$ & $\begin{array}{l}\text { Pseudomonas } \\
\text { aeruginosa }\end{array}$ & CEF & New EVD & 14 & No & Enterococcus spp & $\mathrm{AMP}+\mathrm{CEF}$ & $\begin{array}{l}\text { New EVD } \rightarrow \\
\text { Removed EVD }\end{array}$ & Yes \\
\hline \multirow[t]{2}{*}{10} & M & 67 & $\begin{array}{l}\mathrm{DM}, \mathrm{SICH}, \mathrm{VPS}, \\
\text { craniotomy, EVD }\end{array}$ & $\begin{array}{l}\text { Acinetobacter } \\
\text { baumannii }\end{array}$ & MEP & $\begin{array}{l}\text { Removed VPS, } \\
\text { new EVDs }\end{array}$ & 41 & Fever & $\begin{array}{l}\text { Staphylococcus } \\
\text { aureus }^{\S}\end{array}$ & VA & Removed EVD & No \\
\hline & \multicolumn{12}{|c|}{ Gram $(-) \rightarrow$ G (-) } \\
\hline $11 \mathrm{~A}$ & M & 45 & $\begin{array}{l}\text { Brain tumor, SICH, } \\
\text { craniectomy, VPS }\end{array}$ & $\begin{array}{l}\text { Serratia } \\
\text { marcescens }^{@}\end{array}$ & MEP & $\begin{array}{l}\text { Removed VPS, } \\
\text { new EVD }\end{array}$ & 29 & $\begin{array}{l}\text { Fever, reduced } \\
\text { CSF glucose }\end{array}$ & $\begin{array}{l}\text { Stenotrophomonas } \\
\text { maltophilia }\end{array}$ & MOX+TMP & $\begin{array}{l}\text { Removed EVD } \rightarrow \\
\text { new EVD }\end{array}$ & Treated \\
\hline $11 \mathrm{~B}$ & & & & & & & 90 & No & Candida glabrata & $\mathrm{FLU}+\mathrm{AMB}$ & & Treated \\
\hline $11 \mathrm{C}$ & & & & & & & 48 & Fever & $\begin{array}{l}\text { Stenotrophomonas } \\
\text { maltophilia* }\end{array}$ & $\mathrm{TMP}+\mathrm{TIG}$ & & No \\
\hline 12 & M & 25 & $\begin{array}{l}\text { TICH, craniectomy, } \\
\text { EVD }\end{array}$ & $\begin{array}{l}\text { Pseudomonas } \\
\text { aeruginosa }\end{array}$ & CAZ & New EVDs & 37 & No & $\begin{array}{l}\text { Pseudomonas } \\
\text { aeruginosa + ESBL- } \\
\text { Proteus mirabilis }\end{array}$ & MEP & Removed EVD & Yes \\
\hline
\end{tabular}


Table 1 Clinical data and implicated pathogens in the super-infections of the study patients with adult bacterial meningitis (Continued)

\begin{tabular}{|c|c|c|c|c|c|c|c|c|c|c|c|c|}
\hline 13 & $M$ & 25 & $\begin{array}{l}\text { TICH, craniotomy, } \\
\text { VPS }\end{array}$ & $\begin{array}{l}\text { Pseudomonas } \\
\text { putida }\end{array}$ & CAZ & $\begin{array}{l}\text { Removed VPS, } \\
\text { new EVD }\end{array}$ & 23 & Leukocytosis & Acinetobacter spp $p^{@}$ & $\mathrm{MEP}+\mathrm{SBM}$ & $\begin{array}{l}\text { Removed EVD } \rightarrow \\
\text { New EVDs } \rightarrow \text { VPS }\end{array}$ & Yes \\
\hline 14 & M & 64 & $\mathrm{SICH}, \mathrm{VPS}$ & Serratia marcescens & $V A+M E P$ & $\begin{array}{l}\text { Removed VPS } \rightarrow \\
\text { new EVD }\end{array}$ & 8 & $\begin{array}{l}\text { Fever, altered } \\
\text { consciousness, } \\
\text { hydrocephalus }\end{array}$ & $\begin{array}{l}\text { Acinetobacter } \\
\text { baumannili }\end{array}$ & $\begin{array}{l}\text { MEP+SBM+ } \\
\mathrm{TIG}+\mathrm{CST}\end{array}$ & $\begin{array}{l}\text { Removed EVD } \rightarrow \\
\text { new EVDs } \rightarrow \text { VP } \\
\text { shunt }\end{array}$ & Yes \\
\hline $15 \mathrm{~A}$ & $\mathrm{~F}$ & 71 & $\begin{array}{l}\text { Old meningitis, } \\
\text { hydrocephalus, VPS }\end{array}$ & $\begin{array}{l}\text { Morganella } \\
\text { morganii }\end{array}$ & CAZ & $\begin{array}{l}\text { Externalization } \\
\text { VPS } \rightarrow \text { removed } \\
\rightarrow \text { new VPS }\end{array}$ & 47 & Fever & Proteus mirabilis ${ }^{@}$ & MEP & Removed VPS & Treated \\
\hline $15 B$ & & & & & & & 40 & Fever & $\begin{array}{l}\text { Glucose non- } \\
\text { fermenting bacilli@ }\end{array}$ & $\mathrm{MEP}+\mathrm{SBM}$ & $\begin{array}{l}\text { Scalp debridement } \\
\rightarrow \text { VPS }\end{array}$ & Yes \\
\hline 16 & M & 31 & $\begin{array}{l}\text { TICH, craniotomy, } \\
\text { EVD (removed) }\end{array}$ & $\begin{array}{l}\text { Stenotrophomonas } \\
\text { maltophilia }\end{array}$ & CAZ & New EVDs & 60 & Fever & $\begin{array}{l}\text { Stenotrophomonas } \\
\text { maltophilia }+ \\
\text { Pseudomonas } \\
\text { aeruginosa }\end{array}$ & $\mathrm{MEP}+\mathrm{CIP}$ & $\begin{array}{l}\text { New EVDs } \rightarrow \text { VPS } \\
\rightarrow \text { removed VPS } \rightarrow \\
\text { Omaya implant }\end{array}$ & Yes \\
\hline & Oth & & & & & & & & & & & \\
\hline 17 & $\mathrm{~F}$ & 58 & $\mathrm{SICH}, \mathrm{EVD}$ & $\begin{array}{l}\text { Staphylococcus } \\
\text { epidermidis }{ }^{\S}+ \\
\text { Acinetobacter } \\
\text { Iwoffii }\end{array}$ & $\begin{array}{l}\text { VA+ROC+ } \\
\text { CM }\end{array}$ & $\begin{array}{l}\text { Removed EVD, } \\
\text { new EVD }\end{array}$ & 28 & No & $\begin{array}{l}\text { Staphylococcus } \\
\text { epidermidis }{ }^{\S} \\
\text { Pseudomonas } \\
\text { aeruginosa }\end{array}$ & CAZ & $\begin{array}{l}\text { Removed EVD } \rightarrow \\
\text { New EVD } \rightarrow \text { VPS }\end{array}$ & Yes \\
\hline $18 \mathrm{~A}$ & M & 48 & $\begin{array}{l}\text { TICH, craniectomy, } \\
\text { EVD (removed) }\end{array}$ & Escherichia coli & MEP & $\begin{array}{l}\text { New EVDs } \rightarrow \\
\text { craniotomy }\end{array}$ & 36 & $\begin{array}{l}\text { Scalp wound } \\
\text { infection }\end{array}$ & Candida tropicalis & FLU & $\begin{array}{l}\text { removed EVD, } \\
\text { debridement }\end{array}$ & Treated \\
\hline $18 \mathrm{~B}$ & & & & & & & 9 & No & $\begin{array}{l}\text { Escherichia colit } \\
\text { Acinetobacter } \\
\text { bauamnniit } \\
\text { Enterococcus faecalis }\end{array}$ & AMP & $\begin{array}{l}\text { Debridement } \rightarrow \\
\text { new EVD }\end{array}$ & Treated \\
\hline $18 \mathrm{C}$ & & & & & & & 11 & Fever & $\begin{array}{l}\text { Staphylococcus } \\
\text { aureus }+ \\
\text { Acinetobacter } \\
\text { baumannii }\end{array}$ & $V A+I P M$ & $\begin{array}{l}\text { Removed EVD, new } \\
\text { EVD }\end{array}$ & Treated \\
\hline $18 \mathrm{D}$ & & & & & & & 14 & Fever & $\begin{array}{l}\text { Stenotrophomonas } \\
\text { maltophilia }\end{array}$ & CIP & $\begin{array}{l}\text { Removed EVD, new } \\
\text { EVD } \rightarrow \text { removed }\end{array}$ & Yes \\
\hline 19 & M & 58 & Head injury & $\begin{array}{l}\text { Viridan } \\
\text { streptococci }\end{array}$ & $V A+R O C$ & & 8 & $\begin{array}{l}\text { Hydrocephalus, } \\
\text { ventriculitis, } \\
\text { CSF pleocytosis }\end{array}$ & $\begin{array}{l}\text { Prevotella+ Veilonella } \\
+ \text { Fusobacterium }\end{array}$ & $\begin{array}{l}\text { VA+ROC+ } \\
\text { MET }\end{array}$ & New EVD & No \\
\hline \multicolumn{13}{|c|}{ Less than 7 days interval } \\
\hline 20 & $\mathrm{~F}$ & 64 & $\begin{array}{l}\text { DM, VHD, SICH, } \\
\text { craniectomy, EVD }\end{array}$ & $\begin{array}{l}\text { Escherichia colit } \\
\text { Enterococcus } \\
\text { faecalis }\end{array}$ & $V A+C A Z$ & $\begin{array}{l}\text { Removed EVD, } \\
\text { New EVD }\end{array}$ & 3 & $\begin{array}{l}\text { Fever, } \\
\text { leukocytosis, } \\
\text { CSF pleocytosis }\end{array}$ & $\begin{array}{l}\text { Enterobacter } \\
\text { cloacae }{ }^{\# @}\end{array}$ & $\mathrm{VA}+\mathrm{CAZ}$ & $\begin{array}{l}\text { Removed EVD } \rightarrow \\
\text { VPS }\end{array}$ & No \\
\hline 21 & M & 49 & $\begin{array}{l}\text { Liver cirrhosis, SICH, } \\
\text { craniectomy, EVD } \\
\text { (removed) }\end{array}$ & $\begin{array}{l}\text { Coagulase- } \\
\text { negative } \\
\text { staphylococci }\end{array}$ & VA & New EVD & 5 & No & $\begin{array}{l}\text { Enterobacter } \\
\text { aerogenes }\end{array}$ & CEF & New EVDs & No \\
\hline
\end{tabular}

Oxacillin-resistant; "change antibiogram during treatment; ${ }^{\circledR}$ cephalosporin-resistant; *the time interval from the finding of previous pathogen(s) to the detection of the following new pathogen(s).

Abbreviations: $\mathrm{M}$, male; $\mathrm{F}$, female; EVD, external ventricular drainage; $\mathrm{SICH}$, spontaneous intra-cranial hemorrhage; DM, diabetes mellitus; VPS, ventriculo-peritoneal shunt; TICH, traumatic intra-cranial hemorrhage; ESBL, extended spectrum $\beta$-lactamase; LZD, linezolid; EPM, entrapenem; VA, vancomycin; CAZ, ceftazidime; CEF, cefepime; MEP, meropenem; ROC, ceftraixone; CM, chloroamphenicol; CSF, cerebrospinal fluid; MOX, moxifloxacin; TMP, trimethoprim-sulfamethoxazole; FLU, fluconazole; AMB, amphotericin B; TIG, tigecycline; SBM, sulbactam; ATM, aztreonam; CST, colistin; AMP, ampicillin; MET, metronidazole 
hydrocephalus in 3 episodes (Cases 2, 14, 19), altered consciousness in 2 episodes (Cases 5,14 ), seizure in 1 episode (Case 5), scalp wound infection in 1 episode (Case 18) and appearance of ventriculitis in 1 episode (Case 19). The other 7 episodes without new clinical presentations were detected by regular CSF check-up studies.

Results of antibiotic susceptibilities of the implicated pathogens are listed in Table 2. Initial antibiotic use and the adjusted antibiotic after identification of the pathogens in the super-infections are listed in Table 1. The choices were guided by results of antimicrobial susceptibility tests. In addition to medical treatment, the other 20 patients except Case 8 had undergone removal of EVD or VPS and insertion of a new EVD or VPS.

With medical treatment, 14 patients survived while 7 died. Four of the 14 survivors (Cases 2, 6, 7, and 15) had clear consciousness while the other 10 had disturbed consciousness. One of the four survivors with clear consciousness (Case 7) had full recovery while the other three had quadriparesis. With GOS assessment, Case 7 had good prognosis, while the other 20 had poor prognosis. Comparative results between the fatal and non-fatal groups showed no significant clinical factors (Table 3).

\section{Discussion}

There is an increasing number of ABM patients with preceding neurosurgical condition, according to previous epidemiologic studies [4,14-16]. A preceding neurosurgical state is associated with mixed, relapsing, or recurrent infectious state of $A B M[3,4,8]$. In the current study, all $21 \mathrm{ABM}$ patients with super-infection had a neurosurgical condition as the preceding event and all had undergone a procedure involving the insertion of a neurosurgical device during the entire clinical course. This may imply that neurosurgical device insertion is an important event in the development of super-infection. Aside from this pre-condition, 69\% (18/26) of the superinfection episodes occurred despite an antibiotic-treatment course for bacterial meningitis longer than two weeks (Table 1). Thus, the occurrence of super-infection during $\mathrm{ABM}$ treatment may also suggest an inadequate infectious control program of the hospital.

Due to the preceding neurosurgical condition and existing bacterial meningitis, the clinical presentation of super-infection in ABM may be obscured. In the 27 episodes of the $21 \mathrm{ABM}$ patients in this study, new evidence of meningitis is noted in $74.1 \%(20 / 27)$. Recurrence of fever was the most common, occurring in $63.0 \%(17 / 27)$ of the episodes. Other new evidences of meningeal infection included CSF pleocytosis, peripheral leukocytosis, and altered consciousness. All of these presentations are not unique and can be found in other infectious states, including central nervous system infections [6]. Therefore, a high index of suspicion is important because thus far, only repeat CSF follow-up studies and bacterial culture are the most important steps to confirm the diagnosis of $\mathrm{ABM}$ with super-infection.

Staphylococcal species were the most common G(+) pathogens implicated in both initial $A B M$ and $A B M$ with super-infection in the 21 study patients (Table 1 ). This finding is consistent with previous reports [17-19] that have shown the high prevalence of staphylococcal infection in this specific group of patients. Most of the implicated staphylococcal pathogens shown in this study were methicillin-resistant. However, the presence of these resistant $G(+)$ strains is not a therapeutic problem because intravenous vancomycin is one of the empiric antibiotics for ABM treatment in the hospital $[4,16]$.

It is known that early and appropriate antimicrobial use is of strategic importance in managing ABM $[4,5,7]$. Early diagnosis of super-infection in the therapeutic course is also important because most patients need an adjustment of antimicrobial agents after identification of the newly-implicated pathogens (Table 1). Such adjustments are important in Cases 1-10 because of the conversion from $G(+)$ to $G(-)$ bacterial pathogens in Cases 1-8 and from $G(-)$ to $G(+)$ pathogens in Cases 9 and 10 .

Another important therapeutic consideration in antibiotic use is the emergence of multi-drug resistant bacterial strains in G(-) ABM [11-13,20-25]. This problem is notable especially in patients with underlying $G(-) A B M$ and a super-infection caused by another G(-) pathogen. Case 12 had ESBL-Proteus mirabilis infection, Cases 13 and 14 had multi-drug resistant (MDR) Acinetobacter spp. infections, and Case 16 had ceftazidime- and cefepime-resistant Pseudomonas aeruginosa infection. All four cases needed antibiotic adjustments for the corresponding newly-cultured bacterial pathogens. The emergence of Stenotrophomonas maltophilia as the implicated pathogen was noted in Cases 2, 11, and 18. Its emergence has caused a therapeutic challenge because it is usually not susceptible to traditional antibiotics used in ABM treatment [24,25]. Presently, trimethoprime-sulfamethoxazole or quinolones are the antimicrobial agents of choice [23,24].

In this study, another observed problem is the change of antibiotic susceptibilities during the therapeutic course of super-infection by G(-) pathogens (Table 2). MDR Stenotrophomonas maltophilia-strain emerged in Case 2 and meropenem-resistant A. Baumannii strain in Case 3. Other resistant strains that emerged during the therapeutic course of super-infection were ceftazidimeresistant $A$. baumannii in Case 6, ceftazidime-resistant Pseudomonas aeruginosa in Cases 9 and 12, and ceftazidime-resistant Enterobacter cloacae in Case 20. All of these changes in antibiotic susceptibilities required an adjustment of antimicrobial agents. In the super- 
Table 2 Antibiogram of implicated pathogens in the super-infections of the study patients with adult bacterial meningitis $(\mathbf{n}=\mathbf{2 1})$

\begin{tabular}{|c|c|c|c|c|c|c|c|c|c|}
\hline Case & Pathogens & ROC & CAZ & MAX & MEP & CIP & TIG & TMP-SMX & AMP- SBM \\
\hline 1 & ESBL-Escherichia coli & $\mathrm{R}$ & $\mathrm{R}$ & $\mathrm{R}$ & $\mathrm{s}$ & & $\mathrm{S}$ & & \\
\hline \multirow[t]{2}{*}{2} & Stenotrophomonas maltophilia & & & & & $S$ & & $\mathrm{R}$ & \\
\hline & & & & & & $\mathrm{R}$ & & $\mathrm{R}$ & \\
\hline \multirow[t]{2}{*}{3} & Acinetobacter baumannii & & I & $\mathrm{S}$ & $S$ & $\mathrm{R}$ & & & $S$ \\
\hline & & & $\mathrm{S}$ & $\mathrm{S}$ & $\mathrm{R}$ & $\mathrm{R}$ & & & $\mathrm{S}$ \\
\hline \multirow[t]{2}{*}{4} & Enterobacter cloacae & & $S$ & & $S$ & $S$ & & & \\
\hline & Pseudomonas aeruginosa & & $\mathrm{S}$ & $S$ & $S$ & $\mathrm{~S}$ & & & \\
\hline 5 & Enterobacter cloacae & S & $\mathrm{S}$ & & $S$ & $\mathrm{~S}$ & & & \\
\hline \multirow[t]{2}{*}{6} & Acinetobacter baumannii & & $S$ & $S$ & $S$ & $S$ & & & \\
\hline & & & । & $S$ & $S$ & $S$ & & & \\
\hline 7 & Acinetobacter spp & । & $S$ & $S$ & $S$ & $S$ & & & $S$ \\
\hline 8 & Acinetobacter spp & $\mathrm{S}$ & $S$ & $S$ & $S$ & $S$ & & & $S$ \\
\hline \multirow[t]{2}{*}{9} & Pseudomonas aeruginosa & & $S$ & $S$ & $S$ & $S$ & & & \\
\hline & & & $\mathrm{R}$ & $S$ & $S$ & & & & \\
\hline 10 & Acinetobacter baumannii & & $S$ & $S$ & $S$ & $S$ & & & \\
\hline \multirow[t]{2}{*}{$11 \mathrm{~A}$} & Serratia marcescens & 1 & $\mathrm{R}$ & $\mathrm{R}$ & $\mathrm{S}$ & & & & \\
\hline & Stenotrophomonas maltophilia & & & & & $\mathrm{S}$ & & $\mathrm{S}$ & \\
\hline $11 \mathrm{C}$ & Stenotrophomonas maltophilia & & & & & $\mathrm{R}$ & & $\mathrm{S}$ & \\
\hline \multirow[t]{3}{*}{12} & Pseudomonas aeruginosa & & $\mathrm{S}$ & $S$ & $S$ & $\mathrm{~S}$ & & & \\
\hline & & & $\mathrm{R}$ & $S$ & $\mathrm{~S}$ & $\mathrm{R}$ & & & \\
\hline & ESBL-Proteus mirabilis & $\mathrm{R}$ & $\mathrm{R}$ & $S$ & $S$ & $\mathrm{R}$ & & & \\
\hline \multirow[t]{2}{*}{13} & Pseudomonas putida & & $S$ & $S$ & $\mathrm{R}$ & $S$ & & & \\
\hline & Acinetobacter spp & $\mathrm{R}$ & $\mathrm{R}$ & $\mathrm{R}$ & I & $\mathrm{R}$ & $\mathrm{s}$ & & 1 \\
\hline \multirow[t]{2}{*}{14} & Serratia marcescens & $S$ & $S$ & $S$ & $S$ & $S$ & & & \\
\hline & Acinetobacter baumannii & & $\mathrm{R}$ & $\mathrm{R}$ & $\mathrm{R}$ & $\mathrm{R}$ & S & & $\mathrm{R}$ \\
\hline \multirow[t]{2}{*}{$15 \mathrm{~A}$} & Morganella morganii & $\mathrm{R}$ & $S$ & $S$ & $S$ & S & & & \\
\hline & Proteus mirabilis & $\mathrm{R}$ & $\mathrm{R}$ & $\mathrm{R}$ & $\mathrm{S}$ & $\mathrm{R}$ & & & \\
\hline $15 B$ & Glucose non-fermenting bacilli & $\mathrm{R}$ & $\mathrm{R}$ & $\mathrm{R}$ & $\mathrm{R}$ & 1 & & & \\
\hline \multirow[t]{2}{*}{16} & Stenotrophomonas maltophilia & & & & & $\mathrm{S}$ & & $\mathrm{S}$ & \\
\hline & Pseudomonas aeruginosa & & $\mathrm{R}$ & $\mathrm{R}$ & $S$ & $\mathrm{~S}$ & & & \\
\hline \multirow[t]{2}{*}{17} & Acinetobacter Iwoffii & & $S$ & & $S$ & $S$ & & & \\
\hline & Pseudomonas aeruginosa & & $\mathrm{S}$ & & $S$ & $\mathrm{~S}$ & & & \\
\hline $18 \mathrm{~A}$ & Escherichia coli & & $\mathrm{R}$ & S & $\mathrm{S}$ & & & & \\
\hline $18 \mathrm{~B}$ & Acinetobacter baumannii & & $\mathrm{R}$ & I & $\mathrm{S}$ & $\mathrm{R}$ & & & $\mathrm{R}$ \\
\hline $18 \mathrm{D}$ & Stenotrophomonas maltophilia & & & & & $S$ & & & \\
\hline \multirow[t]{2}{*}{19} & Escherichia coli & S & $S$ & $S$ & S & & & & \\
\hline & Enterobacter cloacae & S & $S$ & $S$ & S & & & & $\mathrm{R}$ \\
\hline \multirow[t]{2}{*}{21} & Enterobacter aerogenes & 1 & I & $S$ & $S$ & $S$ & & & \\
\hline & & $\mathrm{R}$ & $\mathrm{R}$ & $S$ & $\mathrm{~S}$ & $\mathrm{~S}$ & & & \\
\hline
\end{tabular}

Abbreviations: ROC, ceftriaxone; CAZ, ceftazidime; MAX, cefepime; MEP, meropenem; CIP, ciprofloxacin; AMP-SBM, ampicillin-subactam; TIG, tigecycline; TMP-SMX, trimethoprime-sulfamethoxazole; LEV, levofloxacin; ESBL, extended spectrum $\beta$-lactamase; R, resistant; I, intermediate; S, susceptible

Note: ESBL-producing Escherichia coli was suspected by the disk-diffusion susceptibility test [10].

Non-Cephalosporin-susceptible Gram-negative bacteria (GNB) were used to describe isolates that were non-susceptible to ceftriaxone, ceftazidime, and cefepime. Intermediate and resistant isolates were considered non-susceptible [11]. Multi-drugs resistant (MDR) GNB was used to describe isolates that were nonsusceptible to all antibiotics routinely tested including amikacin, (ampicillin-sulbactam in A. baumannii), ceftriaxone, ceftazidime, cefepime, ciprofloxacin, imepenem, and meropenem [12,13]. The antimicrobial susceptibilities TMP-SMX, ciprofloxacin, levofloxacin, and moxifloaxcin of the Stenotrophomonas maltophilia strains isolated from the cerebrospinal fluid were determined concomitantly by the broth dilution method as described in the Clinical and Laboratory Standards (CLSI) guidelines. 
Table 3 Clinical comparison between the fatal and nonfatal groups of the 21 adult bacterial meningitis patients with super-infection

\begin{tabular}{|c|c|c|c|}
\hline Factors & $\begin{array}{l}\text { Non-fatal } \\
(n=14)\end{array}$ & $\begin{array}{l}\text { Fatal } \\
(n=7)\end{array}$ & $P$ \\
\hline Age, median (range) & $48(25-73)$ & $55(41-67)$ & 0.400 \\
\hline \multicolumn{4}{|l|}{ Gender } \\
\hline Male & 8 & 5 & 0.656 \\
\hline Female & 6 & 2 & \\
\hline \multicolumn{4}{|l|}{ Diabetes mellitus } \\
\hline Yes & 1 & 2 & 0.247 \\
\hline No & 13 & 5 & \\
\hline \multicolumn{4}{|l|}{ Presence of new symptoms } \\
\hline Yes & 10 & 5 & 1.000 \\
\hline No & 4 & 2 & \\
\hline \multicolumn{4}{|l|}{ Fever } \\
\hline Yes & 10 & 4 & 0.638 \\
\hline No & 4 & 3 & \\
\hline \multicolumn{4}{|l|}{ Group } \\
\hline $\mathrm{G}(+)>\mathrm{G}(-)$ or $\mathrm{G}(+)>\mathrm{G}(+)$ & 7 & 3 & 1.000 \\
\hline $\mathrm{G}(-) \rightarrow \mathrm{G}(-)$ & 5 & 1 & \\
\hline \multicolumn{4}{|l|}{ Intracranial hemorrhage $(\mathrm{ICH})$} \\
\hline Spontaneous ICH & 4 & 4 & 0.085 \\
\hline Traumatic ICH & 6 & 0 & \\
\hline
\end{tabular}

Abbreviation: G(+), Gram-positive pathogen; G(-), Gram-negative pathogen

infection of Case 18, anaerobic pathogens appeared and metronidazole was administered thereafter.

The appearance of anaerobic pathogens may also pose a therapeutic challenge because metronidazole is not a regular antimicrobial agent used in ABM treatment [3-7]. Other complicated super-infections in $A B M$ were noted in Cases 11 and 18, in which Candida spp was the implicated pathogen. This uncommon super-infection was also noted in the report of Gelfand et al. [2]. Chronic use of broad spectrum antimicrobial agents is an important factor known to encourage the colonization of Candida organisms. Antifungal agents should be used for such an uncommon super-infection in treating ABM.

With an effort of management, the therapeutic result of super-infection ABM showed that $95.2 \%(20 / 21)$ of the involved patients belonged to the poor prognosis group. The mortality rate of this specific group of ABM patients is high $(33.3 \%, 7 / 21)$ and survivors also have a high incidence of severe morbidity. Because most patients with super-infection ABM have both a preceding neurosurgical event and a pre-existing bacterial meningitis, it is difficult to assess the therapeutic outcome of this uncommon infectious state.

\section{Conclusions}

Super-infection in ABM is usually seen in patients with preceding neurosurgical event, including insertion of an external ventricular drainage device. Fever recurrence is the most common presentation and most new evidences are non-specific. Repeat CSF culture study is needed for diagnostic confirmation. The occurrence of super-infection in $A B M$ has a great impact on the $A B M$ treatment because a high percentage of the implicated bacteria strains are non-susceptible to common antibiotics used and early detection is important for adjusting the antibiotic regimen. Infections by unusual pathogens like anaerobes and fungi may appear and these deserve special attention because the choice of antimicrobials will be different. Because the pre-existing neurosurgical condition is complicated by super-infection, prognosis of super-infection in ABM remains poor. The interpretation of clinical data shown in this study is limited by its small case series and the retrospective data collection in a single hospital-based study. Further large-scale study is needed for better delineation of the presentation and prognosis.

\section{Acknowledgements \\ None of the authors are founded in this study. \\ Author details \\ ${ }^{1}$ Department of Neurology, Kaohsiung Chang Gung Memorial Hospital and Chang Gung University College of Medicine, Kaohsiung, Taiwan. ${ }^{2}$ Department of Biological Science, National Sun Yat-Sen University, Kaohsiung, Taiwan. ${ }^{3}$ Neurosurgery, Kaohsiung Chang Gung Memorial Hospital and Chang Gung University College of Medicine, Kaohsiung, Taiwan. ${ }^{4}$ Diagnostic Pathology, Kaohsiung Chang Gung Memorial Hospital and Chang Gung University College of Medicine, Kaohsiung, Taiwan.}

\section{Authors' contributions}

All authors have read and approved the final manuscript. $\mathrm{CRH}$ contributed to the conception and design, data acquisition and analysis, and drafting and revision the manuscript; SFC, CHL, YCC, NWT, CCC, HCW and CCC contributed to the conception and design, and clinical data analysis; and WNC contributed to the conception and design, data analysis, and critical revision and final approval of the revised manuscript.

\section{Competing interests}

The authors declare that they have no competing interests.

Received: 22 January 2011 Accepted: 18 May 2011

Published: 18 May 2011

\section{References}

1. Finland M, Barnes MW: Acute bacterial meningitis at Boston City Hospital during 12 selected years, 1935-1972. J Infect Dis 1977, 136:400-415.

2. Gelfand MS, McGee ZA, Kaiser AB, Tally FP, Moses J: Candidal meningitis following bacterial meningitis. South Med J 1990, 83:567-570.

3. Durand ML, Calderwood SB, Weber DJ, Miller SL, Southwick FS, Caviness VS, Swartz MN: Acute bacterial meningitis in adults: a review of 493 episodes. N Engl J Med 1993, 328:21-28.

4. Chang WN, Lu CH, Huang CR, Tsai NW, Chuang YC, Chang CC, Chen SF, Chien CC: Changing epidemiology of adult bacterial meningitis in southern Taiwan: a hospital-based study. Infection 2008, 36:15-22.

5. Chaudhurl A, Martinez-Martin P, Kennedy PG, Andrew Seaton R, Portegies P, Bojar M, Steiner I, EFNS Task Force: EFNS guidelines on the management of community-acquired bacterial meningitis: report of an EFNS Task Force on acute bacterial meningitis in older children and adults. Eur J Neurol 2008, 15:649-659.

6. Ziai WC, Lewin JJ III: Update in the diagnosis and management of central nervous system infections. Neurol Clin North Am 2008, 26:427-468. 
7. Fitch MT, van de Beek D: Emergency diagnosis and treatment of adult meningitis. Lancet Infect Dis 2007, 7:191-200.

8. Chang WN, Lu CH, Huang CR, Chuang YC: Mixed infection in adult bacterial meningitis. Infection 2000, 28:8-12.

9. Jannett $\mathrm{B}$, Bond $\mathrm{M}$ : Assessment of outcome after severe brain damage. Lancet 1975, 1(7905):480-484

10. Hsieh CJ, Shen YH, Hwang KP: Clinical implications, risk factors and mortality following community-onset bacteremia caused by extendedspectrum $\beta$-lactamase (ESBL) and non-ESBL producing Escherichia coli. $J$ Microbiol Immunol Infect 2010, 43:240-248.

11. Chang CJ, Ye JJ, Yang CC, Huang PY, Chiang PC, Lee MH: Influence of third-generation cephalosporin resistance on adult in-hospital mortality from post-neurosurgical bacterial meningitis. J Microbiol Immunol Infect 2010, 43:301-309.

12. Pena C, Suarez C, Tubau F, Juan C, Moya B, Dominguez MA, Oliver A, Pujol M, Ariza J: Nosocomial outbreak of a non-cefepime-susceptible ceftazidime-susceptible Pseudomonas aeruginosa strain over-expression MexXY-OprM and producing an integron-borne PSE-1 beta-lactamase. Clin Microbiol 2009, 47:2381-2387.

13. Chen SF, Chang WN, Lu CH, Chuang YC, Tsai HH, Tsai NW, Chang WN, Lee PY, Chien CC, Huang CR: Adult Acinetobacter meningitis and its comparison with non-Acinetobacter bacterial meningitis. Acta Neurol Taiwan 2005, 14:125-129.

14. Lu CH, Chang WN, Chang HW: Adult bacterial meningitis in southern Taiwan: epidemiologic trend and prognostic factors. J Neurol Sci 2000 182:36-44

15. Kim BN, Pelep AY, Lodise TP, Lipman J, Li J, Nation R, Paterson DL: Management of meningitis due to antibiotic-resistant Acinetobacter species. Lancet Infect Dis 2009, 9:245-255.

16. Chang WN, Lu CR, Huang CR, Chuang YC, Tsai NW, Chen SF, Chang CC, Wang HC, Chien CC, WU JJ: Epidemiology of adult staphylococcal meningitis in southern Taiwan: a clinical comparison of Staphylococcus aureus infection with coagulase-negative staphylococcal infection. Jpn J Infect Dis 2007, 60:262-266.

17. Chang WN, Lu CH, Wu JJ, Chang HW, Tsai YC, Chen FT, Chien CC: Staphylococcus aureus meningitis in adults: a clinical comparison of infections caused by methicillin-resistant and methicillin-sensitive strains. Infection 2001, 29:245-250.

18. Huang CR, Lu CH, Wu JJ, Chang HW, Chien CC, Lei CB, Chang WN: Coagulase-negative staphylococcal meningitis: clinical characteristics and therapeutic outcomes. Infection 2005, 33:56-60.

19. Tsai MH, Lu CH, Huang CR, Chuang YC, Tsai NW, Tsai HH, Chen SF, Li CS, Chang HW, Chien CC, Chang WN: Bacterial meningitis in young adults in southern Taiwan: clinical characteristics and therapeutic outcomes. Infection 2006, 34:2-8.

20. Hsueh PR, Teng LJ, Chen CY, Chen WH, Yu CJ, Ho SW, Luh KT: Pan drugresistant Acinetobacter baumannii causing nosocomial infections in a university hospital, Taiwan. Emerg Infect Dis 2002, 8:827-832.

21. Kim BN, Peleg AY, Lodise TP, Lipman J, Li J, Nation R, Paterson DL: Management of meningitis due to antibiotic-resistant Acinetobacter species. Lancet Infect 2009, 9:245-255.

22. Huang CR, Lu CH, Chuang YC, Tsai NW, Chang CC, Chen SF, Wang HC, Chien CC, Chang WN: Adult Pseudomonas aeruginosa meningitis: high incidence of underlying medical and/or post-neurosurgical conditions and high mortality rate. Jpn J Infect Dis 2007, 60:397-399.

23. Huang $C R$, Lu CH, Chang WN: Adult Enterobacter meningitis: a high incidence of co-infection with other pathogens and frequent association with neurosurgical procedures. Infection 2001, 29:75-79.

24. Looney WJ, Narita M, Mühlemann K: Stenotrophomonas maltophilia: an emerging opportunist human pathogen. Lancet Infect Dis 2009, 9:312-323.

25. Nicodemo AC, Garcia Paez Jl: Antimicrobial therapy for Stenotrophomonas maltophilia infections. Eur J Clin Microbiol Infect Dis 2007, 26:229-237.

\section{Pre-publication history}

The pre-publication history for this paper can be accessed here: http://www.biomedcentral.com/1471-2334/11/133/prepub

doi:10.1186/1471-2334-11-133

Cite this article as: Huang et al:: Clinical characteristics and therapeutic outcomes of nosocomial super-infection in adult bacterial meningitis. BMC Infectious Diseases 2011 11:133.

\section{Submit your next manuscript to BioMed Central and take full advantage of:}

- Convenient online submission

- Thorough peer review

- No space constraints or color figure charges

- Immediate publication on acceptance

- Inclusion in PubMed, CAS, Scopus and Google Scholar

- Research which is freely available for redistribution 